\title{
Pulmonary administration of remdesivir in the treatment of COVID-19
}

\author{
Henrik Berg Rasmussen, ${ }^{1,6}$ Peter Riis Hansen, ${ }^{2}$ Olivier Taboureau, ${ }^{3}$ Ragnar Thomsen, ${ }^{4}$ and Gesche Jürgens ${ }^{5}$
}

Received 19 July 2020; accepted 2 September 2020; published online 17 September 2020

We read, with great interest, the commentary "Remdesivir for Treatment of COVID-19: Combination of Pulmonary and IV Administration May Offer Additional Benefit" by Sun [1]. In this commentary, Sun [1] suggests that intravenous administration of remdesivir is unlikely to produce sufficiently high concentrations of its active antiviral agent, the nucleoside triphosphate (Nuc-TP), in human lungs to effectively eliminate SARS-CoV-2. This led Sun [1] to propose investigations into pulmonary delivery of remdesivir, modifications of its prodrug moiety, and use of nanoformulations of this agent to improve treatment of COVID-19 pneumonia. We consider that these issues are highly relevant but are uncertain about the conclusion that intravenous administration of remdesivir in the treatment of COVID-19 is unlikely to produce therapeutically effective concentrations of this prodrug and its active antiviral agent in human lungs.

First, results from in vitro assays for assessment of the activity of antiviral agents vary substantially being highly dependent on viral quantification method, viral isolate, and cell type used for viral propagation [2, 3]. Sun [1] retrieved $50 \%$ and $90 \%$ maximal inhibitory concentrations $\left(\mathrm{IC}_{50}\right.$ and $\left.\mathrm{IC}_{90}\right)$ of 0.77 and $1.76 \mu \mathrm{M}$, respectively, for the anti-SARS-CoV-2 activity of remdesivir in Vero E6 African green monkey kidney cells from a study by Wang et al. [4]. Assuming a 10-fold intracellular accumulation of Nuc-TP relative to extracellular remdesivir, these inhibitory values were used to derive the intracellular Nuc-TP $\mathrm{IC}_{50}$ and $\mathrm{IC}_{90}$ values of 7.7 and $17.6 \mu \mathrm{M}$, which were compared with an estimated intracellular Nuc-TP concentration in the range of 4 to $10 \mu \mathrm{M}$ in human lungs [1]. However, other studies have reported $\mathrm{IC}_{50}$ and $\mathrm{IC}_{90}$ values for the anti-SARS-CoV-2 activity of remdesivir that differed from

\footnotetext{
${ }^{1}$ Institute of Biological Psychiatry, Mental Health Centre Sct. Hans, Roskilde, Denmark.

${ }^{2}$ Cardiology Department, Herlev and Gentofte Hospital, Hellerup, Denmark.

${ }^{3}$ Université de Paris, INSERM U1133, CNRS UMR 8251, 75006, Paris, France.

${ }^{4}$ Section of Forensic Chemistry, Department of Forensic Medicine, Faculty of Health Sciences, University of Copenhagen, Copenhagen, Denmark.

${ }^{5}$ Clinical Pharmacology Unit, Roskilde Hospital, Roskilde, Denmark.

${ }^{6}$ To whom correspondence should be addressed. (e-mail: henrik.berg.rasmussen@regionh.dk)
}

those adopted by Sun. This includes the study by Pruijssers et al. [5] that reported $\mathrm{IC}_{50}$ values of 0.001 and $0.009 \mu \mathrm{M}$ in primary human airway epithelial (HAE) cells in two independent experiments, whereas a higher $\mathrm{IC}_{50}$ value of $0.28 \mu \mathrm{M}$ and an $\mathrm{IC}_{90}$ value of $2.48 \mu \mathrm{M}$ were observed with Calu-32B4 respiratory epithelial cells. Pruijssers et al. [5] also found that remdesivir inhibited SARS-CoV2 with $\mathrm{IC}_{50}$ and $\mathrm{IC}_{90}$ values of 1.65 and $2.40 \mu \mathrm{M}$, respectively, in Vero E6 cells, which are highly permissive to SARS-CoV-2 and therefore commonly used to study this virus $[6,7]$. Moreover, an $\mathrm{IC}_{50}$ value of $0.38 \mu \mathrm{M}$ has been reported for the anti-SARS-CoV-2 activity of remdesivir in Caco-2 colorectal adenocarcinoma cells [8]. The differences in in vitro antiviral activity of remdesivir between different cell types may partially reflect differences in the ability to convert this prodrug to its antiviral active agent with the Vero E6 cell line apparently activating remdesivir less efficiently than other types of cells [5]. Hence, this cell line, and probably other Vero cell lines as well, may be less suitable for studies of the intracellular pharmacology of remdesivir. Assuming that Nuc-TP in general accumulates 10 -fold intracellularly relative to extracellular remdesivir, the estimated intracellular $\mathrm{IC}_{50}$ values of Nuc-TP in the above cells would all fall below the intracellular Nuc-TP concentration range of 4 to $10 \mu \mathrm{M}$ in human lungs except for those found using Vero E6 cells. Importantly, the primary HAE cells, in which markedly low $\mathrm{IC}_{50}$ values were produced, may represent a biologically more appropriate in vitro model than established cell lines such as Vero E6. Taken together, the intracellular $\mathrm{IC}_{50}$ and $\mathrm{IC}_{90}$ values of the Nuc-TP in human lungs estimated by Sun [1] appear excessively high as they were based on findings done using the Vero E6 cell line.

Second, the achievable maximum plasma concentration $\left(\mathrm{C}_{\max }\right)$ of remdesivir administered intravenously at an approved dose has been reported to exceed two out of four $\mathrm{IC}_{50}$ values and two out of three $\mathrm{IC}_{90}$ values determined for this prodrug [3]. These $\mathrm{IC}_{50}$ and $\mathrm{IC}_{90}$ values had all been estimated using Vero cell lines for propagation of SARS-CoV-2. Accordingly, comparison of $\mathrm{C}_{\max }$ of remdesivir with the $\mathrm{IC}_{50}$ values for its antiSARS-CoV-2 activity in primary HAE cell cultures would have produced markedly higher $\mathrm{C}_{\max } / \mathrm{IC}_{50}$ ratios and suggested that this prodrug effectively eliminates SARS-CoV-2 in human lungs at approved intravenous doses. 
In brief, the most recent data, which may not all have been available to Sun [1] at the time of the submission of his commentary, do not convincingly support the notion that intravenous administration of remdesivir is unable to produce sufficiently high local concentrations of the active antiviral agent of this prodrug to eliminate SARS-CoV-2 from the lungs in COVID-19 pneumonia. However, this does not exclude that there is a marked potential for improvement of the clinical outcome of COVID-19 pneumonia by combining intravenous and pulmonary administration of remdesivir or by other approaches such as redesign of the remdesivir prodrug moiety and use of nanoformulated drug delivery.

\section{REFERENCES}

1. Sun D. Remdesivir for treatment of COVID-19: combination of pulmonary and IV administration may offer additional benefit. AAPS J. 2020;22:77.

2. Buss N, Cammack N. Measuring the effectiveness of antiretroviral agents. Antivir Ther. 2001;6:1-7.

3. Arshad U, Pertinez H, Box H, Tatham L, Rajoli RKR, Curley P, et al. Prioritization of anti-SARS-Cov-2 drug repurposing opportunities based on plasma and target site concentrations derived from their established human pharmacokinetics. Clin Pharmacol Ther. 2020. https://doi.org/10.1002/cpt.1909.

4. Wang M, Cao R, Zhang L, Yang X, Liu J, Xu M, et al. Remdesivir and chloroquine effectively inhibit the recently emerged novel coronavirus (2019-nCoV) in vitro. Cell Res. 2020;30:269-71.

5. Pruijssers AJ, George AS, Schäfer A, Leist SR, Gralinksi LE, Dinnon KH, et al. Remdesivir inhibits SARS-CoV-2 in human lung cells and chimeric SARS-CoV expressing the SARS-CoV-2 RNA polymerase in mice. Cell Rep. 2020;107940.

6. Barreto-Vieira DF, da Silva MAN, Garcia CC, Miranda MD, Matos AR, Caetano B, et al. Morphology and morphogenesis of SARS-CoV-2 in Vero-E6 cells. Available from: https:// www.researchsquare.com/article/rs-40432/v1. 2020. https:// doi.org/10.21203/rs.3.rs-40432/v1.

7. Ogando NS, Dalebout TJ, Zevenhoven-Dobbe JC, Limpens RWAL, van der Meer Y, Caly L, et al. SARS-coronavirus-2 replication in Vero E6 cells: replication kinetics, rapid adaptation and cytopathology. J Gen Virol. 2020. https://doi.org/10.1099/ jgv.0.001453.

8. De Meyer S, Bojkova D, Cinatl J, Van Damme E, Buyck C, Van Loock M, et al. Lack of antiviral activity of darunavir against SARS-CoV-2. Int J Infect Dis. 2020;97:7-10.

Publisher's Note Springer Nature remains neutral with regard to jurisdictional claims in published maps and institutional affiliations. 